\title{
ECOEFICIENCIA DE LOS MODELOS DE PRODUCCIÓN AGRÍCOLA DE MAÍZ DURO Y SU INFLUENCIA AL CAMBIO CLIMÁTICO EN SHUSHUFINDI ECUADOR
}

\author{
ECO-EFFICIENCY OF THE MODELS OF AGRICULTURAL PRODUCTION OF HARD \\ CORN AND ITS INFLUENCE ON CLIMATE CHANGE IN SHUSHUFINDI ECUADOR \\ Ingrid Estefanía Pinzón Colmenares*(i) y Lenin Javier Ramírez Cando®
}

\author{
Maestría de Investigación en Cambio Climático, Sustentabilidad y Desarrollo. Universidad Andina Simón Bolívar, 170143, Qui- \\ to, Ecuador. \\ *Autor para correspondencia: ipinzong7@gmail.com
}

Manuscrito recibido el 16 de julio de 2019. Aceptado, tras revisión, el 21 de septiembre 2020. Publicado el 1 de marzo de 2021.

\begin{abstract}
Resumen
La ecoeficiencia se define como la cantidad o valor económico de un producto por su influencia ambiental e identifica en términos socioeconómicos y ambientales la sostenibilidad de un sistema. El objetivo de esta investigación fue evaluar la ecoeficiencia de tres modelos de producción agrícola de maíz duro presentes en el cantón Shushufindi, Ecuador. Los modelos identificados en el estudio fueron el modelo convencional (MC), semi-convencional (MS) y tradicional (MT). La influencia ambiental se determinó mediante dos indicadores de impacto contemplados en el Análisis de Ciclo de Vida, como son las emisiones de GEIs, según las directrices del IPCC (2006a) y la huella hídrica (HH), a través de los componentes dados por Hoekstra y col., (2011). Para la evaluación de la ecoeficiencia se consideraron los lineamientos de Ribal y col. (2009), aplicando un modelo de optimización por programación no lineal (GLP). Las emisiones de GEIs del MC fueron de $2926,92 \mathrm{kgCO}_{2} \mathrm{eq} \mathrm{ha}^{-1} \mathrm{año}^{-1}$ y una $\mathrm{HH}$ de $1157,86 \mathrm{~m}^{3}$ ton $^{-1}$, el MS contribuyó con 1209,45 $\mathrm{kgCO}_{2} \mathrm{eq} \mathrm{ha}^{-1} \mathrm{año}^{-1}$ y una $\mathrm{HH}$ de $1201,85 \mathrm{~m}^{3}$ ton $^{-1}$, mientras que las emisiones del MT fueron de $570 \mathrm{kgCO}_{2}$ eq ha ${ }^{-1} \mathrm{año}^{-1}$ y una $\mathrm{HH}$ de $1008,16 \mathrm{~m}^{3}$ ton $^{-1}$. Se determinó que el MT es el modelo más ecoeficiente con un valor de 0,99 . Los resultados permitieron conocer los impactos asociados a los modelos de producción agrícola de maíz y su contribución al Cambio Climático (CC) en ecosistemas sensibles como los que alberga la Amazonía ecuatoriana, para que de esta manera se implementen prácticas agrícolas sostenibles.
\end{abstract}

Palabras clave: Ecoeficiencia, GEIs, huella hídrica, Cambio Climático, maíz. 


\begin{abstract}
Eco-efficiency is defined as the quantity or economic value of a product by its environmental influence, and it identifies the sustainability of a system in socio-economic and environmental aspects. The objective of this research was to evaluate the ecoefficiency of three models of agricultural production of hard corn present in the Shushufindi canton, Ecuador. The models identified in the study were the conventional model (MC), semi-conventional (MS) and traditional (MT). The environmental influence was determined through two impact indicators contemplated in the Life Cycle Analysis, such as GHG emissions, according to the IPCC guidelines IPCC (2006a), and the water footprint (HH) through the components stated by Hoekstra y col., (2011). For the evaluation of ecoefficiency, the guidelines of Ribal y col. (2009) were considered, applying a non-linear programming optimization (LPG) model. GHG emissions per MC were $2926.92 \mathrm{kgCO}_{2} \mathrm{eq} \mathrm{ha}^{-1}$ year $^{-1}$ and an $\mathrm{HH}$ of $1157.86 \mathrm{~m}^{3}$ ton $^{-1}$, MS contributed $1209.45 \mathrm{~kg} \mathrm{CO}_{2} \mathrm{eq} \mathrm{ha}^{-1}$ year ${ }^{-1}$ and a $\mathrm{HH}$ of $1201.85 \mathrm{~m}^{3}$ ton $^{-1}$, while the resulting MT emissions were $570 \mathrm{~kg} \mathrm{CO}_{2}$ eq ha ${ }^{-1}$ year $^{-1}$ and a $\mathrm{HH}$ of $1008.16 \mathrm{~m}^{3} \mathrm{ton}^{-1}$, and it was determined that the MT is the most eco-efficient model with a value of 0.99 . The results allowed to know the impacts associated to the models of agricultural production of maize, its contribution to the Climate Change (CC) in sensitive ecosystems like those of the Ecuadorian Amazon, so that in this way sustainable agricultural practices are implemented.
\end{abstract}

Keywords: Climate Change, greenhouse gases, water footprint, eco-efficiency, corn.

Forma sugerida de citar: Pinzón-Colmenares, I.E. y Ramírez Cando, I.J. (2021). Ecoeficiencia de los modelos de producción agrícola de maíz duro y su influencia al cambio climático en Shushufindi Ecuador.. La Granja: Revista de Ciencias de la Vida. Vol. 33(1):76-91. http://doi.org/ 10.17163/lgr.n33.2021.07.

IDs Orcid:

Ingrid Estefanía Pinzón Colmenares: http: / /orcid.org/000-0002-5963-1021

Lenin Javier Ramírez Cando: http:/ / orcid.org/0000-0002-4855-4796

La Granja: Revista de Ciencias de la Vida 33(1) 2021:76-91.

(C2021, Universidad Politécnica Salesiana, Ecuador. 


\section{Introducción}

Entre las problemáticas que han surgido entorno a la producción agrícola se destaca el impacto ambiental asociado al tipo y dosificación de fertilizantes y fitosanitarios, uso de maquinarias, sistemas de riego, semillas certificadas entre otras, que de una $\mathrm{u}$ otra forman impactan a los recursos agua, suelo y aire. Sin embargo, surgen diversas opciones para establecer mejores prácticas agrícolas que integren los aspectos ambientales y económicos, fomentando la sostenibilidad en la producción agrícola. En este sentido, se origina el concepto de ecoeficiencia, definido por Masuda (2016) como la cantidad o valor del producto por la influencia ambiental "valor económico/impactos ambientales". En el caso de la ecoeficiencia en la agricultura, Rodríguez (2018) conceptualiza a la ecoeficiencia como la capacidad que tiene un sistema de uso de tierra de ser sostenible en términos económicos, sociales y ambientales. En el caso de la ecoeficiencia en la agricultura, Rodríguez (2018) conceptualiza a la ecoeficiencia como la capacidad que tiene un sistema de uso de tierra de ser sostenible en términos económicos, sociales y ambientales. Dentro de este contexto, los gases de efecto invernadero (GEIs) constituyen factores importantes de cambio climático (CC) debido a su potencial de calentamiento global (GWP, por sus siglas en inglés) (IPCC, 2013). El incremento de los GEIs está asociado a las actividades realizadas por los sectores económicos, como es el caso del sector agrícola que ha contribuido con el $24 \%$ de las emisiones globales (IPCC, 2014). El IPCC (2015) reportó que las emisiones provenientes por el sector agrícola a nivel global fueron de $11,76 \mathrm{Gt}_{\mathrm{CO}_{2}}$ eq. Para el caso del Ecuador, las emisiones en el año 2012 fueron de $14512,88 \mathrm{Gg}$ de $\mathrm{CO}_{2}$ eq que corresponden a gases como el $\mathrm{CO}_{2}$, el $\mathrm{CH}_{4}$ y $\mathrm{N}_{2} \mathrm{O}$ procedentes de suelos agrícolas $(46,37 \%)$, fermentación entérica $(43,43 \%)$, cultivo de arroz $(7,48 \%)$, manejo de estiércol $(2,34 \%)$ y la quema de residuos agrícolas $(0,39 \%)$ (MAE, 2017). El incremento de estos GEIs puede provocar graves alteraciones ecológicas y económicas, además de cambios impredecibles en los sistemas climáticos (OMM, 2017).

Considerando que el sector agrícola es uno de los más influyentes en términos de emisiones GEI, para el año 2018 los cultivos transitorios ocuparon el $15,1 \%$ de la superficie total agropecuaria del Ecuador que corresponden a 5,3 millones de hectáreas
(INEC, 2019). Los cultivos transitorios con mayor participación de superficie sembrada son: el maíz duro seco $(40,7 \%)$, el arroz $(32,1 \%)$ y la papa $(2,5 \%)$ (INEC, 2019). El mosaico agropecuario presente en el territorio ecuatoriano ha ido reemplazando ecosistemas nativos como son los páramos y los bosques, como se evidenció entre los años 2008 y 2014, donde la expansión del cultivo de maíz causó la mayor parte del cambio de uso de suelo con el $42 \%$, seguido por el cacao $(15,32 \%)$, la palma africana $(14,5 \%)$ y el café $(11,18 \%)$ (Lasso, 2017). Además, el $80 \%$ del maíz es utilizado como materia prima para la agroindustria en la elaboración de balanceados destinado a la alimentación de animales como aves y porcinos (Baca, 2016).

En Ecuador, la provincia con mayor producción de maíz duro seco es Los Ríos con 38,8\% del total nacional, lo que corresponde a $602 \mathrm{mil} \mathrm{Tm}$ y una superficie sembrada de 383399 ha (INEC, 2019). De acuerdo a los datos de la Encuesta de Superficie y producción Agropecuaria Continua (ESPAC), en el año 2017 la producción de maíz en Los Ríos disminuyó un $4,88 \%$ y se evidenció un incremento de la producción de maíz en provincias que no eran productoras de este grano a gran escala, tal es el caso de la provincia de Sucumbíos que tuvo una superficie total sembrada del 1,99\% de la superficie nacional equivalente a 7732 ha (INEC, 2017). Particularmente, en el cantón Shushufindi, la producción agrícola de maíz está conformada por pequeños y medianos productores dispersos en el territorio cantonal. En el cantón, en parroquias como Shushufindi central, Siete Julio y San Roque se siembran alrededor de 1018 ha de maíz (GAD Shushufindi, 2015).

El maíz se cultiva de manera tradicional en chacra bajo una dinámica de agricultura familiar donde existe la rotación y asociación de cultivos (GADP Limoncocha, 2015). Por otra parte, el modelo productivo comunitario está siendo remplazado por un modelo productivo mecanizado e industrial (Maza, 2015), y está acompañado por paquetes tecnológicos que contienen semillas certificadas e insumos como fertilizantes, herbicidas, insecticidas, además de maquinaria como desgranadoras, cosechadoras de maíz y tractores (GAD Provincial Sucumbíos, 2015; MAG, 2017; GADPR Siete de Julio, 2018). Por todo lo expuesto, los objetivos planteados para la investigación son caracterizar los modelos agrícolas de maíz duro presentes en Shushufindi, estimar las 
emisiones de GEIs y consumo de agua dulce y determinar la ecoeficiencia de los modelos de producción de maíz.

\section{Métodos}

La investigación se basó en los lineamentos planteados por Ribal y col. (2009), el primer paso fue especificar y caracterizar los escenarios o modelos a estudiar. A continuación, se realizó una evaluación ambiental de los modelos mediante el Análisis de Ciclo de Vida (ACV) que contempla dos categorías de impacto: Calentamiento Global (emisiones de GEIs) y consumo de agua dulce (huella hídrica), a su vez, se efectuó una evaluación económica de estos modelos a través del modelo KyK desarrollado por Kuosmanen y Kortelainen. Finalmente, se integraron las dos evaluaciones anteriores utilizando un modelo de programación no lineal GLP (Graphic Linear Optimizer, por sus siglas en inglés) para determinar de esta manera cual modelo tradicional, semiconvencional o convencional es más ecoeficiente en términos socioeconómicos y ambientales.

\subsection{Caracterización e identificación de los modelos:}

Se identificaron tres modelos agrícolas de maíz presentes en la zona de estudio, el modelo convencional (MC), modelo semi-convencional (MS) y el modelo tradicional (MT), estos modelos fueron caracterizados tomando en cuenta los atributos de Martínez (2008) y otros atributos adecuados para el estudio como se presentan en la Tabla 1.

Adicionalmente, los modelos MC, MS y MT fueron georreferenciados con ayuda de un vehículo aéreo no tripulado (UAV por sus siglas en inglés). Posteriormente se procesó la información en un GIS (ArcGIS ${ }^{\circledR}$ ), en el cual se identificó la ubicación y área de las parcelas identificadas para cada modelo (Tabla 2). La distribución geográfica del cultivo de maíz en Shushufindi fue realizada mediante los datos otorgados por el inventario SIPA (Sistema de Información Pública Agropecuaria) (Figura 1).

Una vez identificados los modelos se realizaron entrevistas semiestructuradas a los productores maiceros con el fin de recopilar información para los factores socioeconómico y ambiental, efectuando un muestreo por conveniencia y consecutivo (muestreo no probabilístico). En campo se recolectaron muestras de suelo-raíz y biol para un posterior análisis en el laboratorio. Los parámetros edafológicos analizados en las muestras fueron materia orgánica (MO), carbón orgánico (CO), $\mathrm{pH}$, textura y humedad, y para el caso del biol se analizó Nitrógeno $(\mathrm{N})$, Fósforo $(\mathrm{P})$ y Potasio $(\mathrm{K})$.Todas las muestras fueron analizadas en laboratorios acreditados (LABSU y AGROCALIDAD).

\subsection{Evaluación ambiental}

La influencia ambiental se determinó mediante el Análisis de Ciclo Vida para la agricultura «ACV agrícola» siguiendo la metodología de Arango, Carmona y Romero (2014) y las recomendaciones de Ramírez-Cando y Spugnoli (2016), Oliveral, Cristobal y Saizar (2016), Ramírez-Cando y col. (2017) e IHOBE (2009).

\subsubsection{Límite del sistema a evaluar}

El sistema evaluado se limitó desde la fase de producción agrícola hasta la fase de distribución del maíz. Se incluyen datos de entrada/inputs (recursos, materias primas, insumos, transporte, energía etc.) y salidas/outputs (emisiones al aire (GEIsGWP), agua y suelo, residuos y subproductos) como se presentan en la Figura 2. 
Tabla 1. Caracterización de los modelos de producción de maíz en Shushufindi.

\begin{tabular}{|c|c|c|c|}
\hline & Escenario 1 & Escenario 2 & Escenario 3 \\
\hline $\begin{array}{l}\text { Caracterización } \\
\text { atributo }\end{array}$ & $\begin{array}{l}\text { Modelo convencional } \\
\text { (MC) }\end{array}$ & $\begin{array}{c}\text { Modelo } \\
\text { semi-convencional } \\
\text { (MS) }\end{array}$ & $\begin{array}{l}\text { Modelo tradicional } \\
\text { (MT) }\end{array}$ \\
\hline Energía & $\begin{array}{l}\text { Predomina uso de energía fósil } \\
\text { (gas-petróleo) }\end{array}$ & $\begin{array}{c}\text { Predomina uso de energía } \\
\text { fósil } \\
\text { (gas-petróleo) - o } \\
\text { alternativas (solar) }\end{array}$ & $\begin{array}{l}\text { Uso de energía renovable (solar) } \\
\text { menor predominio de combustibles } \\
\text { fósiles }\end{array}$ \\
\hline Escala & $\begin{array}{l}\text { Área de producción } \\
\qquad>1 \text { ha }\end{array}$ & $\begin{array}{l}\text { Parcelas de } 0,5 \text { ha } \\
\text { Cuadrilla } 0,71 \text { ha } \\
\quad \leq 1 \text { ha }\end{array}$ & $\begin{array}{l}\text { Área de producción } \\
\text { Parcela, chacra }\end{array}$ \\
\hline Objetivo & Comercio & Comercio - autoconsumo & $\begin{array}{c}\text { Autoconsumo - (poco o } \\
\text { nada al comercio) }\end{array}$ \\
\hline $\begin{array}{c}\text { Fuerza de } \\
\text { trabajo }\end{array}$ & Asalariada - Jornal & Jornal - Familiar - mingas & Familiar - Comunal \\
\hline Diversidad & $\begin{array}{c}\text { Monocultivo } \\
\text { Baja diversidad }\end{array}$ & $\begin{array}{c}\text { Monocultivo - Asociados } \\
\text { Baja diversidad }\end{array}$ & $\begin{array}{c}\text { Policultivo } \\
\text { Alta Diversidad }\end{array}$ \\
\hline Productividad & $\begin{array}{c}\text { "Irregular en el tiempo, con alta } \\
\text { productividad laboral; baja } \\
\text { productividad ecológica } \\
\text { y energética" } \\
\text { (Martínez, 2008). }\end{array}$ & $\begin{array}{c}\text { "Irregular en el tiempo, con alta } \\
\text { productividad laboral; baja } \\
\text { productividad ecológica } \\
\text { y energética" } \\
\text { (Martínez, 2008). }\end{array}$ & $\begin{array}{l}\text { "Regular en el tiempo. } \\
\text { Alta productividad } \\
\text { ecológico - energética; } \\
\text { baja productividad en } \\
\text { el trabajo" } \\
\text { (Martínez, 2008). }\end{array}$ \\
\hline Semilla & $\begin{array}{l}\text { Modificada, } \\
\text { Híbrido }\end{array}$ & Curada & Criollo \\
\hline $\begin{array}{l}\text { Maquinaria } \\
\mathbf{y} \\
\text { herramientas }\end{array}$ & $\begin{array}{c}\text { Maquinaria agrícola } \\
\text { de gran tamaño } \\
\text { (Desgranadora, cosechadora, } \\
\text { canguro agrícola, guadaña) }\end{array}$ & $\begin{array}{c}\text { Maquinaria agrícola } \\
\text { artesanal } \\
\text { (Desgranadora artesana - } \\
\text { guadaña) }\end{array}$ & $\begin{array}{c}\text {-No existe uso de } \\
\text { maquinaria agrícola } \\
\text {-Para el } \\
\text { presente estudio } \\
\text { el productor } \\
\text { utilizó guadaña }\end{array}$ \\
\hline Insumos & $\begin{array}{l}\text {-Fitosanitarios } \\
\text {-Fertilizantes } \\
\text { sintéticos }\end{array}$ & $\begin{array}{l}\text {-Fitosanitarios } \\
\text {-Fertilizantes } \\
\text { sintéticos }\end{array}$ & $\begin{array}{l}\text {-Abonos } \\
\text { orgánicos } \\
\text {-Bioles } \\
\text {-Compost }\end{array}$ \\
\hline $\begin{array}{l}\text { Prácticas } \\
\text { agrícolas }\end{array}$ & -Sin rotación de cultivos & $\begin{array}{c}\text {-Puede o no } \\
\text { existir la rotación } \\
\text { de cultivos }\end{array}$ & -Rotación de cultivos \\
\hline $\begin{array}{l}\text { Presencia de } \\
\text { plagas }\end{array}$ & $\mathrm{Si}$ & $\mathrm{Si}$ & $\mathrm{Si}-\mathrm{No}$ \\
\hline $\begin{array}{c}\text { Control de } \\
\text { plagas }\end{array}$ & Agroquímico & Agroquímico & $\begin{array}{l}\text { Control } \\
\text { natural }\end{array}$ \\
\hline Desechos & $\begin{array}{l}\text {-Reincorporación } \\
\text { de residuos, } \\
\text { quema } \\
\text {-Desechos } \\
\text { de } \\
\text { agroquímicos } \\
\end{array}$ & $\begin{array}{l}\text {-Reincorporación } \\
\text { de residuos, } \\
\text { quema } \\
\text {-Desechos de } \\
\text { agroquímicos }\end{array}$ & $\begin{array}{l}\text {-Reincorporación } \\
\text { de residuos } \\
\text {-Producción } \\
\text { de desechos } \\
\text { orgánicos }\end{array}$ \\
\hline Conocimiento & $\begin{array}{l}\text { Especializado, } \\
\text { ciencia, } \\
\text { convencional, } \\
\text { estandarizado }\end{array}$ & Local - Convencional & $\begin{array}{c}\text { Local, } \\
\text { tradicional basado } \\
\text { en creencias de } \\
\text { transmisión limitada. } \\
\text { Y conocimientos } \\
\text { permaculturales }\end{array}$ \\
\hline Cosmovisión & $\begin{array}{c}\text { Mercadocéntrica: } \\
\text { "la naturaleza es un sistema } \\
\text { separado de la sociedad, } \\
\text { cuyas riquezas deben } \\
\text { ser explotadas a través } \\
\text { de la ciencia y la técnica" } \\
\text { (Martínez, 2008). }\end{array}$ & Mercadocéntrica & $\begin{array}{c}\text { Ecocéntrica: } \\
\text { "La naturaleza es } \\
\text { una entidad viva y sacral. } \\
\text { Lo natural se encarna } \\
\text { en deidad con quien debe } \\
\text { dialogar durante la } \\
\text { apropiación" } \\
\text { (Martínez, 2008). }\end{array}$ \\
\hline
\end{tabular}

Fuente: (Martínez, 2008) -observaciones en campo y entrevistas semiestructuradas. 
ZONA N WGS $84-18 \mathrm{~S}$

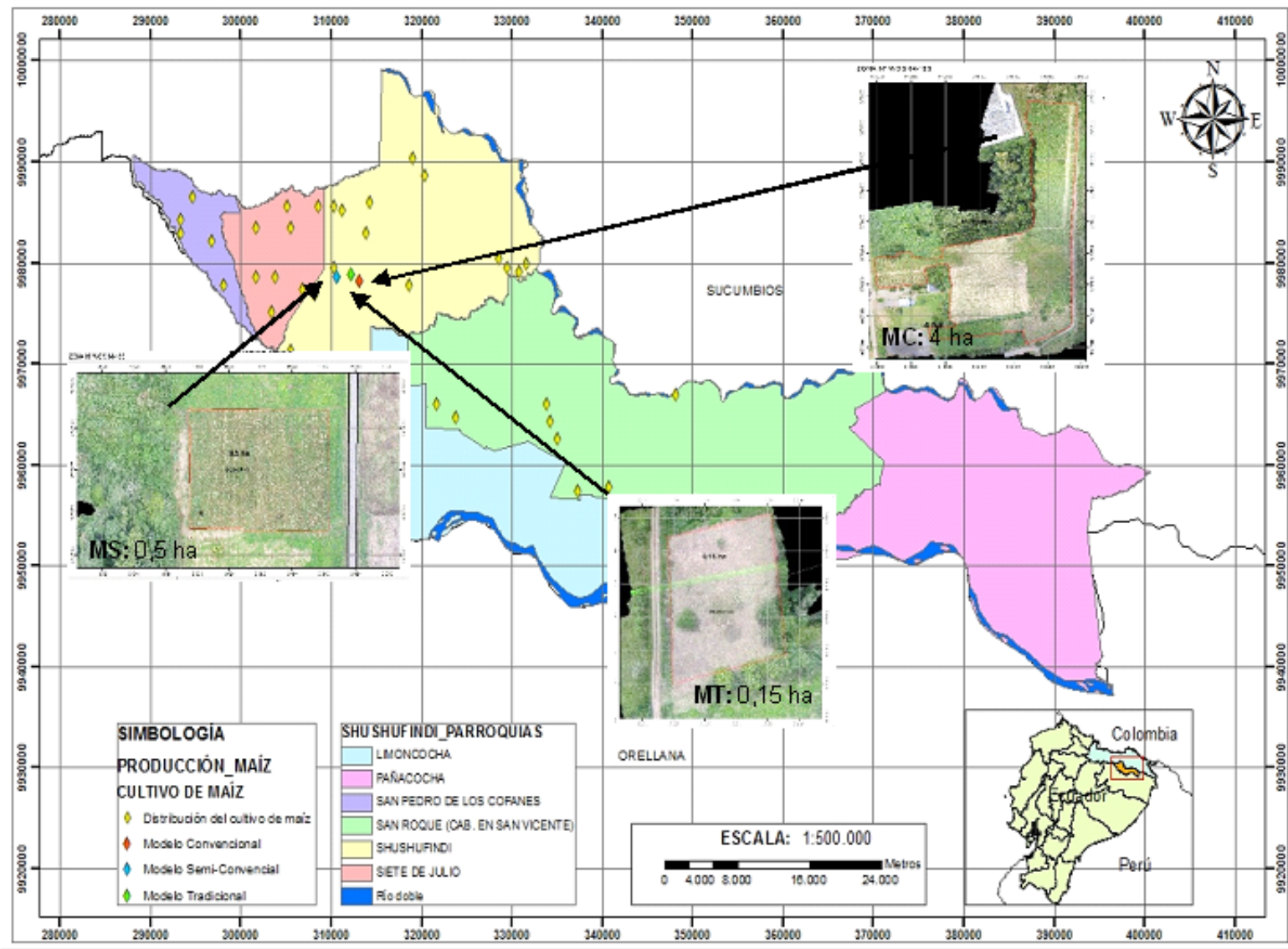

Figura 1. Distribución geográfica de los cultivos de maíz en las parroquias de San Pedro de los Cofanes, Siete de Julio, San Roque, Shushufindi cabecera cantonal, y la ubicación de las parcelas de estudio para cada modelo MC, MS y MT. Fuente: Datos GPS essential, georreferenciación de parcelas drone Phantom 4, Sistema Nacional Información (SNI), (GAD Shushufindi, 2015)

Tabla 2. Descripción del área y ubicación de las parcelas de investigación por cada modelo estudiado

\begin{tabular}{|c|c|c|c|c|c|}
\hline \multirow{2}{*}{$\begin{array}{c}\begin{array}{c}\text { Tipo de } \\
\text { Modelo de } \\
\text { producción }\end{array} \\
(\mathrm{MC}) \\
\end{array}$} & \multicolumn{2}{|c|}{$\begin{array}{c}\text { Área } \\
\text { muestreada }\end{array}$} & \multicolumn{3}{|c|}{ Ubicación } \\
\hline & 4 & ha & 310599 & 9978558 & Vía Atahualpa \\
\hline (MS) & 0,5 & ha & 310662 & 9978571 & $\begin{array}{l}\text { Los Ríos-Terreno } \mathrm{N}^{\circ} 11 \\
\text { Lotización de la asociación } \\
\text { de empleados de } \\
\text { Shushufindi. }\end{array}$ \\
\hline (MT) & 0,15 & ha & 312274 & 9978832 & $\begin{array}{c}\text { Vía Shushufindi- Finca } \\
\text { RICAAMA** }^{*}\end{array}$ \\
\hline
\end{tabular}

* Coordenadas de ubicación de las parcelas de estudio en UTM WGS 84 18S

** RICAAMA: Riqueza del campo amazónico - finca permacultural. 


\subsubsection{Categorías de impacto}

En el estudio se consideraron dos categorías de impacto: calentamiento global ( $\mathrm{kg}$ equivalentes de $\mathrm{CO}_{2}$ ) y el consumo de agua ( $\mathrm{m}^{3} /$ ton), estimado a través de la huella hídrica.

\section{a) Emisiones de GEIs}

Para estimar las emisiones de GEIs se consideraron las directrices del IPCC 2006 para el sector AFOLU (sector de agricultura, silvicultura y otros usos de la tierra por sus siglas en inglés) (IPCC, 2006a; IPCC, 2006b). Adicionalmente, se siguieron las recomendaciones del protocolo de GEIs (WRI \& WBCSD, 2011) y los lineamientos tomados de Agri-footprint (Durlinger y col., 2017a; Durlinger y col., 2017b). Cabe señalar que para el estudio se consideraron las fuentes de emisiones (E) de GEIs que contemplan un impacto mayor al $1 \%$ de acuerdo al criterio de IPCC "cut-off", como se presenta a continuación:

- E por el uso de combustibles y lubricantes.

- E por fertilizantes (orgánicos- sintéticos) $\mathrm{N}, \mathrm{P}, \mathrm{K}$.

- E de $\mathrm{CO}_{2}$ por la aplicación de urea.

- E de $\mathrm{N}_{2} \mathrm{O}$ por el $\mathrm{N}$ aplicado en suelos gestionados.

- E por la aplicación de fitosanitarios.

- E por la semilla de maíz.

- E por la quema de residuos agrícolas.

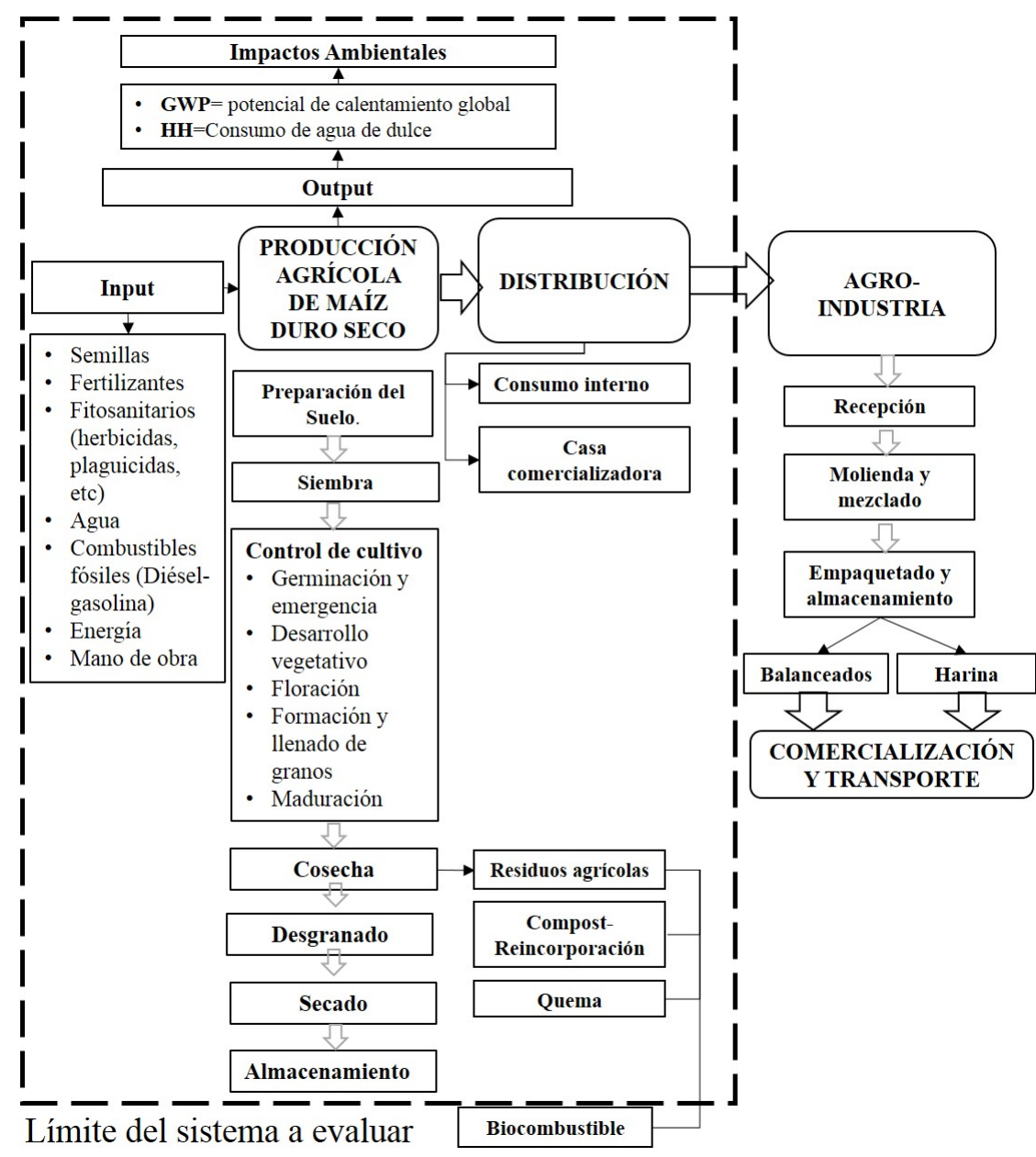

Figura 2. Límite del sistema evaluado - ACV agrícola del maíz duro. El sistema evaluado va desde producción de maíz hasta la distribución, se excluyen las fases de la agroindustria, comercialización y transporte. 
La emisiones de GEIs se estimaron mediante la ecuacion general dada por WRI \& WBCSD (2011) (Ecuación 1). Con DA representa la cantidad empleada de un insumo en un lugar durante un periodo de tiempo y en una zona determinada, ejemplo cantidad de combustible usados por el tractor. $F E$ representa los coeficientes que cuantifican las emisiones o absorciones de un gas dependiendo de los datos de actividad. Los factores de emisiones para el estudio fueron los determinados por el IPCC y por el BioGrace (2011). Y GW P es el potencial de Calentamiento Global para los gases de $\mathrm{CO}_{2}$ (1), $\mathrm{CH}_{4}$ (25) y $\mathrm{N}_{2} \mathrm{O}$ (298) para un horizonte temporal de 100 años (IPCC, 2007). Es importante mencionar que para fines comparativos se extrapolaron los datos de actividad de los modelos MT y MS a una hectárea.

$$
\mathrm{kgCO}_{2} e q / h a=D A * F E * G W P
$$

\section{b) Consumo de agua dulce (huella hídrica-HH)}

Para medir la huella hídrica $(\mathrm{HH})$ se consideraron los componentes de huella hídrica verde $\mathrm{HH}_{\mathrm{verde}}$ (precipitación) y huella hídrica gris $\mathrm{HH}_{\text {gris }}$ (contaminación de agua dulce), permitiendo así conocer el volumen total de agua dulce utilizada por los cultivos de maíz en Shushufindi. Es importante mencionar que en el lugar de estudio no se aplica riego debido a las precipitaciones significativas presentes, por lo que la huella hídrica azul $\mathrm{HH}_{\mathrm{azul}}$ (componente de la $\mathrm{HH}$ ) asociada a las precipitaciones no fue evaluada. Mediante la Ecuación 2 (Pérez, 2012) se calculó la HH del cultivo de maíz para cada modelo de producción agrícola estudiado.

$$
H H_{\text {cultivo }}=H H_{\text {verde }}+H H_{\text {gris }}\left(\frac{m^{3}}{\text { ton }}\right)
$$

Finalmente, para el cálculo de la HH se utilizó el programa CROPWAT 8.0 ® desarrollado por la Organización de la Naciones Unidas para la Agricultura y Alimentación (FAO) y tabulaciones en EXCEL, siguiendo los Manuales de Evaluación de la Huella Hídrica de la Water Footprint Network (WFN) y la FAO realizado por Franke, Boyacioglu y Hoekstra (2013).

\subsection{Evaluación económica}

Para la evaluación económica se aplicó el modelo KyK desarrollado por Kuosmanen y Kortelainen y descrito por Ribal y col. (2009). Se consideró la acumulación parcial de los costos a lo largo del proceso de producción agrícola de maíz (semilla, combustibles, fertilizantes, fitosanitarios, jornales, insumos, alquiler de maquinaria) para cada modelo analizado (\$ha/año). Además, se cuantificó la rentabilidad de la producción de maíz mediante las Ecuaciones 3, 4 y 5, planteada por Ayala-Garay y col. (2013).

$$
\begin{gathered}
\text { Rentabilidad }=I T-C T \\
I T=P_{y} Y \\
C T=P_{x} X
\end{gathered}
$$

Donde: IT es el ingreso total $\left(\mathrm{ha}^{-1}\right), C T$ es el costo total de la producción, $P_{y}$ es el precio de mercado del cultivo $\mathrm{Y}$ (\$/ton), $Y$ el rendimiento del cultivo $\left(\right.$ ton $\left.\mathrm{ha}^{-1}\right), P_{x}$ es el precio del insumo o actividad $\mathrm{X}$ (ton $\mathrm{ha}^{-1}$ ) y $X$ es la actividad o insumo.

\subsection{Ecoeficiencia}

La ecoeficiencia se evaluó mediante la ecuación de Rincón y Wellens (2011), como se presenta en la Ecuación 6.

$$
\text { Ecoeficiencia }=\frac{\text { Valor del producto o servicio }}{\text { Influencia ambiental }}
$$

A partir de la Ecuación 6, Ribal y col. (2009), plantean un modelo de programación no lineal para $m$ escenarios (modelos $M C, M S, M T$ ) y $n$ categorías de impacto (emisiones de GEIs-HH) (Ecuaciones 7, $8,9)$. Este cálculo fue efectuado en Microsoft Excel usando el aplicativo Solver.

$\underset{x}{\operatorname{mawE} E} i=\frac{V_{i}}{w_{1} \cdot z_{i 1}+w_{2} \cdot z_{i 2}+\cdots+w_{n} \cdot z_{i n}}$

Con la condición siguiente:

$\frac{V_{1}}{w_{1} \cdot z_{i 1}+w_{2} \cdot z_{i 2}+\cdots+w_{n} \cdot z_{i n}} \leq 1$
$\frac{V_{m}}{w_{1} \cdot z_{m 1}+w_{2} \cdot z_{m 2}+\cdots+w_{n} \cdot z_{m n}} \leq 1$

Y $w_{1}, w_{2}, \ldots, w_{n} \geq 0$. Donde $V_{i}$ es el valor económico añadido del escenario $i=1 \ldots, m$ (\$/ton), $w_{i}$ es el peso del impacto ambiental $j=1, \ldots n, z_{i j}$ representa el impacto ambiental (huella gris, huella verde $\mathrm{y}$ GEIs), $j=1, \ldots, n$ por unidad funcional para el escenario $i=1, \ldots, m$. El índice de eco-eficiencia varía entre 0 y 1 , donde el valor 1 indicará que el escenario es eco-eficiente (Ribal y col., 2009). 


\section{Resultados y Discusión}

\subsection{Inputs y outputs}

En la Tabla 3 se presenta el inventario de las inputs y outputs de los modelos evaluados teniendo en cuen- ta los resultados de laboratorio, entrevistas con los productores y obsevaciones en campo.

Tabla 3. Inputs y ouputs de los modelos evaluados

\begin{tabular}{|c|c|c|c|c|c|}
\hline \multicolumn{2}{|c|}{ DESCRIPCIÓN } & \multicolumn{3}{|c|}{ CANTIDAD } & \multirow[t]{2}{*}{ UNIDAD } \\
\hline Inputs* & & MT & MS & MC & \\
\hline \multirow{12}{*}{$\begin{array}{l}\text { Fertilizantes } \\
\text { Sintéticos }\end{array}$} & 10-30-10 & - & 2 & 1088,62 & \multirow{12}{*}{$\mathrm{kg} / \mathrm{ha}$ año ${ }^{-1}$} \\
\hline & $\mathrm{N}$ & - & 0,2 & 108,86 & \\
\hline & $\mathrm{P}$ & - & 0,6 & 326,59 & \\
\hline & $\mathrm{K}$ & - & 0,2 & 108,86 & \\
\hline & $13-40-13$ & - & '- & 2 & \\
\hline & $\mathrm{N}$ & - & - & 0,26 & \\
\hline & $\mathrm{P}$ & - & - & 0,80 & \\
\hline & $\mathrm{K}$ & - & - & 0,26 & \\
\hline & Engrosador & - & 4 & 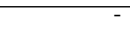 & \\
\hline & $\mathrm{N}$ & & 0,33 & - & \\
\hline & $\mathrm{P}$ & & 0 & - & \\
\hline & $\mathrm{K}$ & & 1,16 & - & \\
\hline \multirow{4}{*}{ Biol } & $\begin{array}{l}\text { Lixiviado de } \\
\text { compost + orina }\end{array}$ & 16 & - & - & $\mathrm{L} / \mathrm{ha}$ año ${ }^{-1}$ \\
\hline & $\mathrm{N}$ & 8,96 & - & - & \multirow{3}{*}{$\mathrm{kg} / \mathrm{ha}$ año ${ }^{-1}$} \\
\hline & $\mathrm{P}$ & 0,35 & - & - & \\
\hline & $\mathrm{K}$ & 2,13 & - & - & \\
\hline & & & & 181,82 & \\
\hline Urea & - & - & & 83,64 & $\mathrm{~kg} / \mathrm{ha}$ año ${ }^{-1}$ \\
\hline Fitosanitarios & & - & 0,43 & 1,25 & $\mathrm{~kg} / \mathrm{ha}$ año ${ }^{-1}$ \\
\hline \multirow{3}{*}{ Combustibles } & Gasolina & 6,28 & 22,75 & 9,46 & \multirow{3}{*}{$\mathrm{gal} / \mathrm{ha} \mathrm{año}^{-1}$} \\
\hline & Diésel & - & - & 29 & \\
\hline & Aceite $2 \mathrm{~T}$ & 0,35 & 0,53 & - & \\
\hline Semilla & & 48,38 & 45,36 & 40 & $\mathrm{~kg} / \mathrm{ha}$ año ${ }^{-1}$ \\
\hline Agua & Riego & 0 & 0 & 0 & $\mathrm{~m}^{3} / \mathrm{ha} \mathrm{año}^{-1}$ \\
\hline Trabajo & & 1366,67 & 552 & 121,68 & h/ha año ${ }^{-1}$ \\
\hline \multicolumn{6}{|l|}{ Outputs $^{* * *}$} \\
\hline Maíz duro & & 3,92 & 2,40 & 6,35 & \\
\hline Residuo agrícola & & 4,49 & 3,16 & 7,28 & ton/ha año ${ }^{-1}$ \\
\hline
\end{tabular}

\subsection{Categoría de calentamiento global}

\subsubsection{Estimación de GEIs de los modelos de produc- ción de maíz.}

El modelo de producción agrícola de maíz que tuvo mayor aportación de GEIs fue el MC con una emisión estimada alrededor de 2926,92 $\mathrm{kg} \mathrm{CO}_{2} \mathrm{eq}$ $\mathrm{ha}^{-1} \mathrm{año}^{-1}$, seguido por las emisiones procedentes del MS que emitió $1209,45 \mathrm{~kg} \mathrm{CO}_{2} \mathrm{eq} \mathrm{ha}^{-1}$ año $^{-1}$, mientras que en el MT las emisiones fueron menores con $570 \mathrm{~kg} \mathrm{CO}_{2} \mathrm{eq} \mathrm{ha}^{-1} \mathrm{año}^{-1}$ (Tabla 4). 
En el presente estudio se evidenció que en el MT las emisiones son $80 \%$ más bajas que las del MC y $57 \%$ menores que las del MS. De igual manera, un estudio realizado por Eranki, Devkota y Landis (2019) reportó que en un escenario de agricultura ecológica las emisiones eran $41 \%$ más bajas que las emisiones emitidas por una agricultura convencional. Asimismo, se reportaron valores de 145,32 (MT), 561,21 (MS) y 460,91 (MC) kgCO $\mathrm{kg}_{2} /$ ton (Figura 4-Tabla 6), comparado con el estudio de Altu- na y col. (2012) donde se determinó que la huella de carbono del maíz fue de $514,76 \mathrm{~kg}$ de $\mathrm{CO}_{2} \mathrm{eq} /$ ton de producto, siendo esta huella mayor que otros cereales como el trigo $\left(380,87 \mathrm{~kg}\right.$ de $\mathrm{CO}_{2} \mathrm{eq} /$ ton $)$ y la cebada $\left(297,75 \mathrm{~kg}\right.$ de $\left.\mathrm{CO}_{2} \mathrm{eq} / \mathrm{ton}\right)$. Es importante mencionar que independientemente del modelo, sea MS y MC las emisiones son mayores que las que se reportaron en Perú por la producción mecanizada de maíz con $224 \mathrm{~kg} \mathrm{CO}_{2}$ eq ton ${ }^{-1}$ (Morales y col., 2018).

Tabla 4. Total de emisiones procedentes de los modelos estudiados

\begin{tabular}{|c|c|c|c|c|}
\hline \multirow{2}{*}{$\begin{array}{c}\text { TOTAL, DE EMISIONES } \\
\text { DE GEIs }\end{array}$} & \multicolumn{3}{|c|}{$\begin{array}{l}\text { modelos de producción } \\
\text { agrícola de maíz duro }\end{array}$} & \multirow[t]{2}{*}{ kg de $\mathrm{CO}_{2}$ eq/ha/año } \\
\hline & MT & MS & MC & \\
\hline $\mathrm{kg}$ de $\mathrm{CO}_{2}$ eq/ha/año & 570,00 & 1209,45 & 2926,92 & \\
\hline$\%$ de aportación de GEIs & 13 & 27 & 60 & \\
\hline \multirow[t]{2}{*}{$\mathrm{kgCO}_{2} \mathrm{eq} /$ ton } & 145,32 & 561,21 & 460,91 & \\
\hline & & & & $=\mathrm{MT}=\mathrm{MS}=\mathrm{MC}$ \\
\hline
\end{tabular}

$\approx \mathrm{MT}=\mathrm{MS} \approx \mathrm{MC}$

$\mathrm{kg} \mathrm{CO}$ eq ton $^{-1}$ año-1

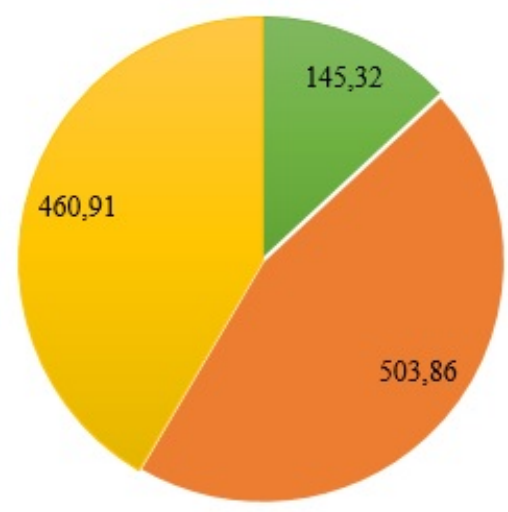

Figura 3. Emisiones de GEIs por los modelos estuadiados $\left(\mathrm{kg} \mathrm{CO}_{2} \mathrm{eq} \operatorname{ton}^{-1}\right.$ año $\left.^{-1}\right)$

\subsubsection{Emisiones procedentes del uso de fertilizantes}

Las emisiones procedentes de la aplicación de fertilizantes con aportes de NPK fueron de 54,26 (MT) y 4,49 (MS) $\mathrm{kg}$ de $\mathrm{CO}_{2}$ eq ha $^{-1} \mathrm{año}^{-1}$ y 1032 (MC), kg de $\mathrm{CO}_{2}$ eq ha ${ }^{-1} \mathrm{año}^{-1}$. Adicionalmente, el MC aportó $133, \mathrm{~kg}$ de $\mathrm{CO}_{2}$ eq año/ha por la fertilización con urea. Abrahão, Carvalho y Causapé (2016) reportaron que la principal fuente de emisiones de GEIs eran por el uso de fertilizantes líquidos (69\%) y en un $18 \%$ por los abonos compuestos, aportando una huella de carbono por la producción de maíz de $1700 \mathrm{~kg}$ de $\mathrm{CO}_{2}$ eq año/ha.

\subsubsection{Emisiones de $\mathrm{N}_{2} \mathrm{O}$}

El uso de fertilizantes sintéticos, orgánicos y la descomposición del rastrojo son responsables de emisiones significativas de $\mathrm{N}_{2} \mathrm{O}$ debido a los pro- 
cesos de nitrificación, desnitrificación, lixiviaciónvolatilización y escurrimientos que se efectúan en el suelo. Estas emisiones fueron de 443,93 (MT), 234,72 (MS) y $1279,81(\mathrm{MC}) \mathrm{kg}$ de $\mathrm{CO}_{2} \mathrm{eq} \mathrm{ha}^{-1} \mathrm{año}^{-1}$; siendo el MC el modelo que presentó mayor aporte de emisiones emitidos a la atmósfera.

\subsubsection{Emisiones procedentes del uso de combustibles fósiles}

Las emisiones por el uso de combustibles fósiles (gasolina, diésel y lubricantes) por parte de la maquinaria agrícola como la guadaña, tractor, cosechadora, desgranadora y el transporte tuvieron un aporte de emisiones a la atmósfera de 406,49, 196,30 y $54,88 \mathrm{~kg} \mathrm{CO}_{2} \mathrm{eq} \mathrm{ha}^{-1}$ año $^{-1}$ para los modelos MC, MS y MT, respectivamente. En la Figura 4 se puede apreciar el porcentaje y los $\mathrm{kg}$ de $\mathrm{CO}_{2} \mathrm{eq} \mathrm{ha}^{-1} \mathrm{año}^{-1}$ emitidos por las maquinarias utilizadas en cada modelo estudiado.

\subsubsection{Emisiones procedentes por uso de fitosanita- rios.}

Los modelos de producción agrícola reportaron emisiones por la aplicación de fitosanitarios de 9,48 $\mathrm{kg}$ de $\mathrm{CO}_{2}$ eq ha ${ }^{-1}$ (MS) y 60,34 kg de $\mathrm{CO}_{2}$ eq ha ${ }^{-1}$ año ${ }^{-1}$ para el modelo $\mathrm{MC}$, mientras que en el modelo MT no se reportaron emisiones procedentes por esta fuente, ya que el productor no aplica ningún tipo de fitosanitario. De acuerdo con la investigación realizada por Morales y col. (2018), el uso de plaguicidas en cultivos de maíz mecanizado contribuyó con $205 \mathrm{kgCO}_{2} \mathrm{eq} \mathrm{ha}^{-1}$ siendo estas mayores que las estimadas en el presente estudio. En la Tabla 5 se presenta el aporte de emisiones de GEIs de cada fitosanitario utilizados por los modelos MC y MS.

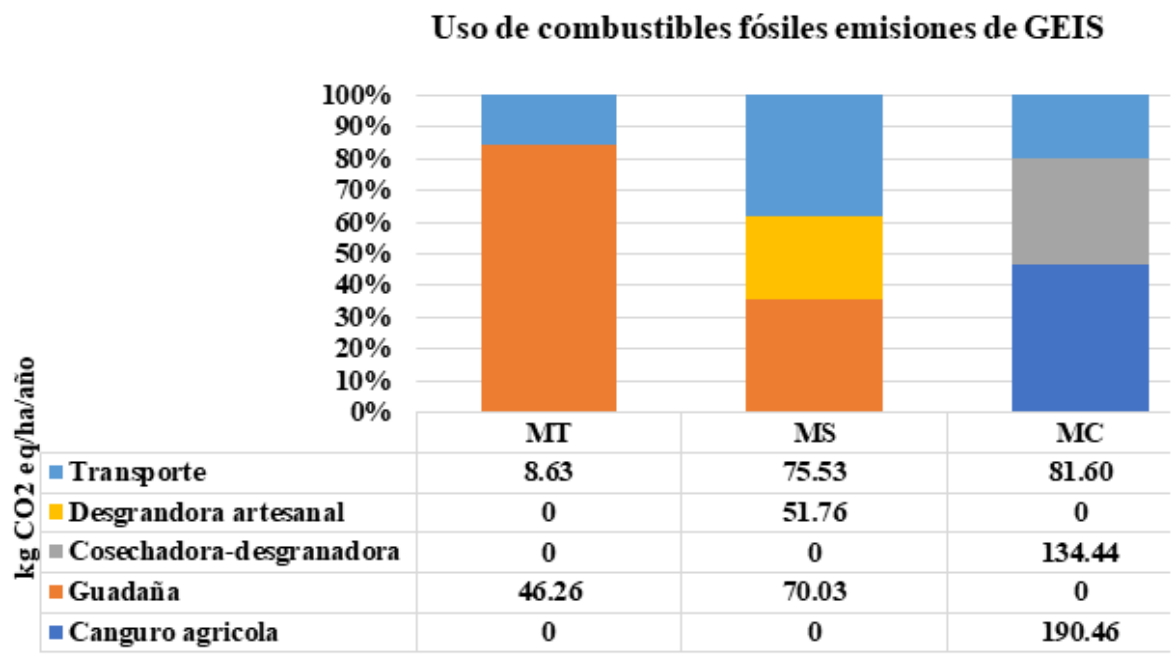

Figura 4. Participación de GEIs por parte de la maquinaria y transporte.

\subsubsection{Emisiones procedentes por el input de semilla}

El uso de semillas contribuyeron con 2,97\%, 1,18\% y $0,48 \%$ para los modelos MT, MS y MC, respectivamente, correspondiente a emisiones de 16,93 (MT) 15,88 (MS) y 14 (MC) kg de $\mathrm{CO}_{2}$ eq ha ${ }^{-1}$ año $^{-1}$, y similares a las reportadas por Abrahão, Carvalho y Causapé (2016), que aportaron un 3\% a las emisiones.

\subsection{Huella hídrica}

\subsubsection{Huella hídrica del cultivo (HHcultivo)}

Las HHcultivo de maíz fueron de 1008,16 (MT), 1201,85 (MS) y 1157,86 (MC) $\mathrm{m}^{3} /$ ton (Figura 5), evidenciando un mayor impacto en los modelos MC y MS, ya que el volumen de agua dulce utilizada directa o indirectamente para producir maíz es muy alto conparado con el MT. En el MS se obtuvo una HHverde $1153,75 \mathrm{~m}^{3} /$ ton, cantidad mayor que la del MT con una HHverde de $1008,16 \mathrm{~m}^{3} /$ ton. El 
modelo convencional a su vez presentó una huella verde de $599,69 \mathrm{~m}^{3} /$ ton, este valor de huella verde dependió del rendimiento (ton/ha) que presenta el modelo convencional comparado entre los otros dos modelos MS y MT.

La $\mathrm{HH}_{\mathrm{verde}}$ del cultivo de maíz en Sucumbíos fue $2073 \mathrm{~m}^{3}$ /ton de acuerdo a Pérez (2012); esta huella es mayor que la estimada en los tres modelos analizados en el presente estudio. Por otra parte, Romero, Quintero y Monserrate (2016) reportaron que la huella verde promedio representa el $60 \%$ de la huella agrícola total $\left(820,24 \mathrm{~m}^{3} /\right.$ ton $)$ en los culti- vos de maíz en Colombia, y la $\mathrm{HH}$ verde representa el $52 \%$ en comparación con el MC.

En cuanto a la huella gris, esta fue de 48,10 y $558,17 \mathrm{~m}^{3} /$ ton para MS y MC, respectivamente. Por su parte, el MT no se reportó valores de $\mathrm{HH}$ gris debido a que no se utilizó ningún tipo de fertilizante sintético ni fitosanitario. En la provincia de $\mathrm{Su}-$ cumbios, Pérez (2012) reportó una HHgris de 330 $\mathrm{m}^{3} /$ ton para el cultivo de maíz, siendo esta menor que la huella gris del MC del presente estudio, debido a que este modelo posee un alto consumo de insumos agrarios como fertilizantes y fitosanitarios.

Tabla 5. Emisiones de GEIs por tipo de fitosanitario

\begin{tabular}{|c|c|c|c|c|c|}
\hline \multirow{2}{*}{\multicolumn{2}{|c|}{ Fitosanitarios }} & $\begin{array}{c}\text { Quemante } \\
\text { (Herbicida) }\end{array}$ & $\begin{array}{c}\text { TEJO } \\
\text { (insecticida) }\end{array}$ & $\begin{array}{c}\text { NOSTOC } \\
\text { (Herbicida) }\end{array}$ & $\begin{array}{r}\text { Total de } \\
\text { emisiones }\end{array}$ \\
\hline & & & $\mathrm{kg}$ de $\mathrm{CO}_{2} \mathrm{eq}$ & $\mathrm{aa}^{-1} \mathrm{año}^{-1}$ & \\
\hline \multirow{2}{*}{ Modelo } & MC & 27,425 & 32,91 & - & 60,34 \\
\hline & MS & - & 8,78 & 0,7 & 9,48 \\
\hline
\end{tabular}

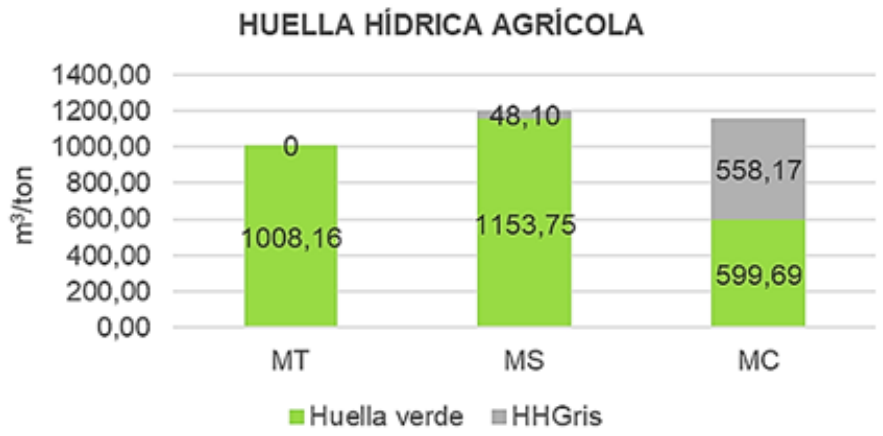

Figura 5. HHcultivo de los modelos de producción de maíz en Shushufundi.

\subsubsection{Evaluación económica}

En la Tabla 6 se presenta el balance de costos por la producción de maíz por modelo estudiado y se visualiza que la producción de maíz es poco rentable por los altos costos que tienen los insumos agrarios, arrojando ganancias muy bajas.

\subsection{Ecoeficiencia}

Los pesos por cada categoría de impacto fueron de 1 para huella gris, 0,41 para la huella verde y 0,92 para las emisiones de GEIs (Tabla 7). Mediante los pesos se obtuvo que el modelo tradicional es el modelo más ecoeficiente en términos ambientales y económicos con 0,99 de ecoeficiencia.La ecoeficiencia está influenciada por los hotspot o puntos críticos que tiene cada categoría de impacto (huella verde, gris y emisiones de GEIs), es por esta razón que la categoría HHgris tiene mayor peso, debido al alto uso de fertilizantes sintéticos y fitosanitarios utilizados por los modelos MS y MC. 
Tabla 6. Balance de costos por la producción de maíz por modelo estudiado.

\begin{tabular}{|c|c|c|c|}
\hline Modelo & MT & $\begin{array}{c}\text { MS } \\
\text { b/ha año }\end{array}$ & 1) \\
\hline Ingresos & 863,00 & 756,00 & 2100,00 \\
\hline Costos & 312,95 & 521,21 & 1712,85 \\
\hline Semilla & 0,00 & 100,00 & 176 \\
\hline Combustibles & 23,45 & 27,71 & 12,35 \\
\hline Fertilizan & 0,00 & 8,00 & 286,00 \\
\hline Fitosanitar & 0,00 & 64,00 & 84,00 \\
\hline Jornales & 270,00 & 240,00 & 540 \\
\hline Ins & 9,50 & 12,50 & 34,50 \\
\hline Alquiler & 10,00 & 69,00 & 580,00 \\
\hline Rentabilidad & 550,05 & 234,79 & 387,15 \\
\hline
\end{tabular}

Para la categoría de emisiones de GEIs, el hotspot está influenciado por el alto consumo de fertilizantes nitrogenados, lo cual genera importantes emisiones de $\mathrm{N}_{2} \mathrm{O}$ por los suelos gestionados. Estos dos componentes duplican su peso (importancia para la gestión aún más eficiente del proceso), respecto de la HHverde, considerando que los GEIs y la HHgris tienen su mayor influencia proveniente del uso de fertilizantes. Este hotspot es el primero que se debe- ría tomar en cuenta para mejorar la ecoeficiencia de los cultivos convencionales o los sistemas combinados de producción de maíz.

Es importante mencionar que la ecoeficiencia es relativa para los modelos estudiados, es decir, que pueden existir otras prácticas agrícolas que haga que los modelos sean más eficientes en términos ambientales y socioeconómicos (Ribal y col., 2009).

Tabla 7. Ecoeficiencia de los modelos de producción de maíz

\begin{tabular}{lccc}
\hline $\begin{array}{c}\text { Categorías de } \\
\text { impacto } \\
(\mathbf{Z})\end{array}$ & HHgris & HHverde & $\begin{array}{c}\text { Emisiones } \\
\text { de GEIs }\end{array}$ \\
\hline Peso $(\mathbf{W})$ & $\mathrm{W}_{1}$ & $\mathrm{~W}_{2}$ & $\mathrm{~W}_{3}$ \\
\multicolumn{4}{c}{0,92} \\
\hline máx. EE & 0,999999617 \\
\hline Ecoeficiencia de los modelos de producción agrícola \\
\hline $\mathbf{E E}_{M S}$ & 0,24 \\
$\mathbf{E E}_{M C}$ & 0,32 & \\
$\mathbf{E E}_{M T}$ & 0,99 \\
\hline
\end{tabular}

\section{Conclusiones}

Mediante el índice de ecoeficiencia se pudo determinar que el modelo de producción agrícola de maíz más sostenible en términos económicos como ambientales es el modelo tradicional MT. El modelo MT presentó una ecoeficiencia de 0,99. Esta alta ecoeficiencia se debe a que este modelo no es dependiente de insumos como fitosanitarios y fertilizantes sintéticos que, a su vez, tienen un alto costo económico. Además, que el uso de estos insumos agroquímicos presenta un impacto ambiental significativo al medio ambiente como se pudo evidenciar en las diferentes categorías de impacto.

A pesar de que el modelo MT es más ecoeficiente es muy poco utilizado por los productores maiceros, debido a que demanda mayor laboriosidad de horas hombres trabajadas y bajo rendimiento (tabla 3) comparado con un modelo convencional (Pinzón 
y Ramírez, 2019); lo que no satisface la demanda económica. En cambio este tipo de modelo MT, es más amigable con el medio ambiente, no depende del paquete tecnológico, en vez de este utiliza semillas criollas no modificadas, ya que, utilizan semilla de la cosecha anterior, no aplican fitosanitarios ni fertilizantes químicos. Por último este modelo preserva una agricultura familiar, prácticas agrícolas sostenibles como la rotación de cultivos para el control de plagas, además de una visión a la permacultura (Pinzón y Ramírez, 2019).

Finalmente, los resultados permitieron conocer los impactos asociados a los modelos de producción agrícola de maíz, su contribución al Cambio Climático (CC) en ecosistemas sensibles como los que alberga la Amazonía ecuatoriana, para que de esta manera se implementen prácticas agrícolas sostenibles.

\section{Agradecimientos}

Nuestro agradecimiento especial a la Universidad Andina Simón Bolívar "UASB", la cual, mediante su apoyo financiero mediante "beca de colegiatura" abrió las puertas para formar profesionalmente a uno de los autores del presente trabajo de investigación.

\section{Referencias}

Abrahão, R., M. Carvalho y J. Causapé (2016). «Carbon and water footprints of irrigated corn and non-irrigated wheat in Northeast Spain». En: Environmental Science and Pollution Research 24.6, 5647-5653. Online: https:/ / bit.ly/3oy214o.

Altuna, A. y col. (2012). «Huella de carbono de los cereales: análisis de la emisión de gases de efecto invernadero en el sector agroalimentario». En: Navarra agraria 194, 31-38. Online:https: / / bit . ly/2LOZV43.

Arango, A., L.G. Carmona y S.A. Romero (2014). "Análisis de ciclo de vida en el sector agrícola:el caso del municipio de Viotá, Cundinamarca (Colombia)». En: Ambiente y Desarrollo 18.35, 117-131. Online: https://bit.ly/3przPCV.
Ayala-Garay, A. y col. (2013). «Análisis de rentabilidad de la producción de maíz en la región de Tulancingo, Hidalgo, México». En: Agricultura, sociedad y desarrollo 10.4, 381-395. Online:https: / / bit.ly/392Bp8v.

Baca, L. (2016). «La producción de maíz amarillo en el Ecuador y su relación con la soberanía alimentaria». Tesis. PUCE.

BioGrace (2011). BioGrace complete list of standard values version Public.

Durlinger, B. y col. (2017a). Agri-footprint 4.0 -Part 1: Methodology and basic principles. Inf. téc. Agrifootprint.

- (2017b). Agri-footprint 4.0 -Part 2: Description of data. Inf. téc. Agri-footprint.

Eranki, P. L., J. Devkota y A. E. Landis (2019). "Carbon footprint of corn-soy-oats rotations in the US Midwest using data from real biological farm management practices». En: Journal of Cleaner Production 210. Elsevier Ltd., 170-180. Online:http:/ / bit.ly/3pARTdR.

Franke, N., H. Boyacioglu y A. Hoekstra (2013). Grey water footprint accounting: Tier 1 supporting guidelines. The Netherlands.

GAD Provincial Sucumbíos (2015). Agricultures de la parroquía San Roque estrenan maquinaría agrícola. Inf. téc. Gobierno Provincial de Sucumbíos.

GAD Shushufindi (2015). Actualización del Plan de Desarrollo y Ordenamiento Territorial del Cantón Shushufindi. Inf. téc. Gobierno Aútonomo Desentralizado de Shushufindi.

GADP Limoncocha (2015). Plan De Desarrollo Y Ordenamiento Territorial De La Parroquia Rural De Limoncocha pp. 1-132. Inf. téc. Gobierno Aútonomo Desentralizado de Limoncocha.

GADPR Siete de Julio (2018). Cosechadora de maiz y arroz en la parroquía Siete de Julio en Shushufindi. Inf. téc. Gobierno Aútonomo Desentralizado de la parroquía Siete de Julio.

IHOBE (2009). Análisis de ciclo de vida y Huella de carbono: Dos maneras de medir el impacto ambiental de un producto. IHOBE S.A. Sociedad Pública de Gestión Ambiental. Vasco.

INEC (2017). Encuesta de superficie y producción agropecuaria continua. Inf. téc. Instituto Nacional de Estadísticas y Censos. Online:https: / / bit.ly / 392SIpZ.

- (2019). Encuesta de Superficie y Producción Agropecuaria Continua (ESPAC) 2018. Inf. téc. Instituto Nacional de Estadísticas y Censos. Online:https: //bit.ly/2YcJYaN. 
IPCC (2006a). «Agricultura, Silvicultura y Otros Usos de la Tierra.» En: Directrices del IPCC 2006 para los Inventarios Nacionales de Gases de Efecto Invernadero. Ed. por S. Eggleston. Vol. 4. IPCC.

- (2006b). «Energía». En: Directrices del IPCC 2006 para los Inventarios Nacionales de Gases de Efecto Invernadero. Ed. por S. Eggleston. Vol. 2. IPCC.

- (2007). Climate Change 2007: Working Group I: The Physical Science Basis- 2.10.2 Direct Global Warming Potentials - AR4 WGI Chapter 2: Changes in Atmospheric Constituents and in Radiative Forcing. Inf. téc. Grupo Intergubernamental de Expertos sobre el Cambio Climático.

- (2013). Cambio Climático 2013: Bases Físicas. Contribución del Grupo de trabajo I al Quinto Informe de Evaluación del Grupo Intergubernamental de Expertos sobre el Cambio Climático. Inf. téc. Online:https:/ / bit.ly/3pdMs4A. Grupo Intergubernamental de Expertos sobre el Cambio Climático.

- (2014). Cambio climático 2014 mitigación del cambio climático. Resumen para responsables de politicas. Inf. téc. IPCC.

- (2015). Cambio climático 2014 informe de síntesis. Inf. téc. Grupo Intergubernamental de Expertos sobre el Cambio Climático.

Lasso, G. (2017). «Territorios en disputa: un análisis de la Soberanía alimentaria en el Ecuador». En: El futuro de la alimentación y retos de la agricultura para el siglo XXI. Vol. 40, pág. 32.

MAE (2017). Tercera Comunicación Nacional del Ecuador a la Convención Marco de las Naciones Unidas sobre el Cambio Climático. Inf. téc. Ministerio de Ambiente del Ecuador.

MAG (2017). MAGAP y GADs de Pañacocha y Shushufindi entregaron kits de insumos de arroz y maíz -Ministerio de Agricultura y Ganadería. Inf. téc. Ministerio de Agricultura y Ganadería. Online:https:/ / bit.ly /398AFil.

Martínez, R. (2008). «Agricultura tradicional campesina: características ecológicas». En: Tecnología en Marcha 21, 3-13.

Masuda, K. (2016). «Measuring eco-efficiency of wheat production in Japan: a combined application of life cycle assessment and data envelopment analysis». En: Journal of Cleaner Production 126, 373-381. Online:https://bit.ly/3c143sH.

Maza, O. (2015). De la Producción Comunitaria a la Producción Mecanizada e Industrial en Limoncocha. Inf. téc. Gobierno Autónomo Descentralizado municipal de Shushufindi.
Morales, R. y col. (2018). «Producción mecanizada de maíz, camote y yuca en la Costa Desértica Peruana: Estimación de la huella de carbono y propuestas de mitigación». En: Ecología Aplicada 17.1, 13-21. Online:https:/ / bit.ly/2LKzZa3.

OMM (2017). BOLETÍN DE LA OMM SOBRE LOS GASES DE EFECTO INVERNADERO- Estado de los gases de efecto invernadero en la atmósfera según las observaciones mundiales realizadas en 2016. Inf. téc. Organización Mundial de la Salud.

Oliveral, A., S. Cristobal y C. Saizar (2016). «Análisis de ciclo de vida ambiental, económico y social». En: INNOTEC Gestión 7, 20-27. Online: https: / / bit.ly/39wBnpS.

Pérez, S (2012). «Evaluación y análisis de la huella hídrica y agua virtual de la producción agrícola en el Ecuador». Tesis de mtría. Zamorano, Honduras: Escuela Agrícola Panamericana.

Pinzón, I. y L. Ramírez (2019). «Influencia de los modelos de producción agrícola de maíz duro al cambio climático en el cantón Shushufindi». Tesis de mtría. Quito, Ecuador: Universidad Andina Simón Bolívar.

Ramírez-Cando, L. y P. Spugnoli (2016). «A review of life cycle assessment: agroproducts modeling». En: La Granja 24.2, 5-15. Online:https: / / bit.ly/3qFOL2g.

Ramírez-Cando, L. y col. (2017). «Environmental assessment of flax straw production for non-wood pulp mills». En: Chemical Engineering Transactions 58. Online:https:/ / bit.ly/2Y2r46d.

Ribal, J. y col. (2009). «Medición de la ecoeficiencia en procesos productivos en el sector agrario: caso de estudio sobre producción de cítricos». En: Economía agraria y recursos naturales 9.2, 125-148. Online:https:/ / bit.ly/39Pci8m.

Rincón, E. y A. Wellens (2011). «Cálculo de indicadores de ecoeficiencia para dos empresas ladrilleras mexicanas». En: Revista internacional de contaminación ambiental 27.4, 333-345. Online:https:/ / bit.ly /3c1VldB.

Rodríguez, E. (2018). «Indicadores de Ecoeficiencia de Sistemas Productivos Agícolas de la Altillanura Plana en la Orinoquia Colombiana». Tesis de mtría. Universidad Nacional de Colombia.

Romero, M., M. Quintero y F. Monserrate (2016). Elementos Técnicos para la Medición de Huella Hídrica en Sistemas Agrícolas. Inf. téc. Colombia: Centro Internacional de Agricultura Tropical (CIAT). 
WRI \& WBCSD (2011). «Greenhouse Gas Protocol. Product Life Cycle Accounting and Reporting Standard, World Resources Institute, Washing- ton DC, USA. Greenhouse Gas Protocol. Online:http:/ / bit.ly /2YtIxog». 